\title{
Spectral polarization and spectral phase control of time-energy entangled photons
}

\author{
Barak Dayan, ${ }^{*}$ Yaron Bromberg, Itai Afek, and Yaron Silberberg \\ Department of Physics of Complex Systems, Weizmann Institute of Science, Rehovot 76100, Israel
}

(Received 16 April 2006; published 4 April 2007)

\begin{abstract}
We demonstrate a scheme to spectrally manipulate a collinear, continuous stream of time and energy entangled photons to generate beamlike, bandwidth-limited fluxes of polarization-entangled photons with nearly degenerate wavelengths. Utilizing an ultrashort-pulse shaper to control the spectral phase and polarization of the photon pairs, we tailor the shape of the Hong-Ou-Mandel interference pattern, demonstrating the rules that govern the dependence of this interference pattern on the spectral phases of the photons. We then use the pulse shaper to generate all four polarization Bell states. The singlet state generated by this scheme forms a very robust decoherence-free subspace, extremely suitable for long-distance fiber-optics-based quantum communication.
\end{abstract}

DOI: 10.1103/PhysRevA.75.043804

PACS number(s): 42.50.Dv, 03.67.Mn, 42.65.Lm, 42.65.Re

Parametrically generated polarization-entangled photons are a primary resource in the fields of quantum communication and quantum information [1], motivating an ongoing search for better means for the generation and control of high fluxes of such photons [2-4]. In particular, the generation of the singlet Bell state has raised a considerable interest due to the fact that it forms a decoherence-free subspace (DFS), which is inherently immune to collective decoherence $[5,6]$. The upper limit for the flux of entangled photon pairs is typically set by the spatial divergence of the down-converted photons, or by the repetition rate of the pump pulses, in the case of pulsed down-conversion. In both cases, the achievable flux is many orders of magnitude lower than the physical upper limit, which is set by the down-converted bandwidth $[7,8]$. Although collinear and continuous downconversion enables efficient generation and collection of photon pairs, resulting in ultrahigh (bandwidth-limited) fluxes as high as $10^{12} \mathrm{~s}^{-1}$ [8], such a single-mode configuration does not readily enable polarization entanglement.

Pulse-shaping techniques were recently introduced as a means to control the spectral properties of broadband downconverted photons [9]. In this work we use a phase and polarization pulse shaper [10] to control both the phase and the polarization of each of the spectral modes of copropagating entangled photons. Our precise control of the phase and polarization in the frequency domain is demonstrated by tailoring the shape of the Hong-Ou-Mandel interference pattern [11]. Exploiting the fact that the photon pairs are hyperentangled, i.e., entangled in more than one degree of freedom [12], we treat the frequency domain as a two-dimensional subspace and thus use the pulse shaper as a Bell-state synthesizer, generating all four polarization Bell states in a collinear, beamlike fashion, which enables bandwidth-limited fluxes, and is suitable for fiber-optic-based quantum communication. The fact that in this scheme the photons of each pair share the same single spatial mode and are nearly degenerate makes it a very robust and practical DFS. Specifically, if the

\footnotetext{
*Present address: Norman Bridge Laboratory of Physics 12-33, California Institute of Technology, Pasadena, California 91125, USA.
}

photons travel through the same fiber, this singlet state is expected to be immune to geometric phase $[13,14]$, birefringence, and all orders of chromatic dispersion, preventing dephasing to distances that can exceed $100 \mathrm{~km}$ (in typical optical fibers).

To illustrate the principles of our scheme, let us consider a collinear, degenerate photon pair in the state $|\varphi\rangle=(1 / \sqrt{2})$ $\times\left(|2\rangle_{H^{-}}-|2\rangle_{V}\right)$, where the subscripts $H, V$ indicate the horizontal and vertical polarizations of the same spatial mode. This state can be generated by a cascaded, collinear type-I down-conversion in two orthogonal crystals [3]. However, this is also the state generated by a collinear type-II downconversion in a single crystal, which emits a pair of photons with orthogonal polarizations $X$ and $Y$, oriented at $45^{\circ}$ to $H, V$ since [4]

$$
|\varphi\rangle=\frac{1}{\sqrt{2}}\left(|2\rangle_{H}-|2\rangle_{V}\right)=|1\rangle_{X}|1\rangle_{Y}
$$

We are specifically interested in the case where the downconverted spectrum is significantly broader than the spectrum of the pump, since this situation implies that the photons are time and energy entangled, having a larger uncertainty in their individual energies than in their collective energy (and the opposite in the time-difference domain). This is typically the case with continuous, degenerate spontaneous down-conversion, especially in the configuration of two type-I crystals (as in our experiment). The state of the photons in this case would therefore be more accurately represented by

$$
|\varphi\rangle=\int_{0}^{\omega_{0}} d v \frac{g(v)}{\sqrt{2}}\left(|1\rangle_{\omega_{0}+v, H}|1\rangle_{\omega_{0}-v, H}-|1\rangle_{\omega_{0}+v, V}|1\rangle_{\omega_{0}-v, V}\right),
$$

where the spectral function $g(v)$ is determined by the nonlinear coupling and the phase-matching conditions in the crystal, and $\omega_{0}=\omega_{p} / 2$, with $\omega_{p}$ being the pump frequency. As is evident from Eq. (2), although the two photons share the same broadband spectrum, they are always on opposite halves of the spectrum, having opposite detunings $\pm v$ from $\omega_{0}$. We therefore use the spectral degree of freedom to dis- 


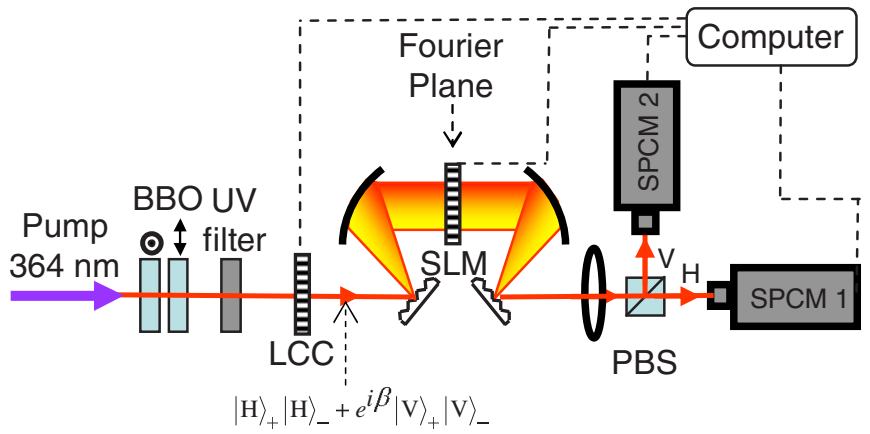

FIG. 1. (Color online) Experimental setup. In addition to an initial harmonic separator filter and final bandpass filters $(70 \mathrm{~nm}$ around $728 \mathrm{~nm}$ ) in front of the SPCMs, gold reflection coatings were used throughout the setup to attenuate the uv pump with respect to the down-converted photons.

tinguish the photons rather than the spatial modes. To clarify the polarization entanglement of $|\varphi\rangle$ let us define two spectral modes by assigning the subscripts,+- to the spectral modes with positive and negative detunings, respectively [15]. Adopting the notation $|H\rangle \equiv|1\rangle_{H}$, we may rewrite Eq. (2) as

$$
|\varphi\rangle=\frac{1}{\sqrt{2}}\left(|H\rangle_{+}|H\rangle_{-}-|V\rangle_{+}|V\rangle_{-}\right) .
$$

Thus, we see that the state $|\varphi\rangle$ is already a polarization Bell state, specifically the state $\left|\phi^{(-)}\right\rangle_{\mathrm{H}, \mathrm{V}}$. This Bell state can be transformed into the Bell state $\left|\psi^{(+)}\right\rangle_{H, V}$ by a simple rotation of $45^{\circ}$, since $\left|\phi^{(-)}\right\rangle_{H, V}$ at the axes $H, V$ is $\left|\psi^{(+)}\right\rangle_{X, Y}$ at the axes $X, Y$. Similarly, the Bell state $\left|\phi^{(+)}\right\rangle_{H, V}$ can be created from $\left|\phi^{(-)}\right\rangle_{H, V}$ by introducing polarization-dependent phase shift (for example, with a birefringent material). However, the singlet state $\left|\psi^{(-)}\right\rangle_{H, V}$ is unique. Having a zero collective "spin," the singlet state forms a DFS, and is not affected by any mechanism that acts equally on both modes; accordingly, it cannot be created by such mechanisms. Since in our case the two modes are the two halves of the down-converted spectrum, in order to create the singlet state one must apply spectrally dependent birefringence. Conveniently enough, this is exactly what a pulse shaper does. A pulse shaper (see Fig. 1) separates the spectral components of the incoming beam by a grating, and focuses them on a spatial-light modulator (SLM), located at the Fourier plane [16]. The SLM is composed of an array of liquid-crystal cells that induce a voltage-controlled birefringence, i.e., a relative phase between the two polarizations of the incoming light. A second set of a curved mirror and a reflection grating performs the inverse Fourier transform and restores the collinear propagation of the beam. Utilizing a pulse shaper, one can apply a phase shift of $\pi$ between the $X$ and $Y$ polarizations only on the upper half of the spectrum (a spectral " $\pi$ step function"). This will create a $\pi$ phase shift between the $|X\rangle_{+}|Y\rangle_{-}$and the $|Y\rangle_{+}|X\rangle_{-}$components of the $\left|\psi^{(+)}\right\rangle_{X, Y}$ state, turning it into the desired singlet state $\left|\psi^{(-)}\right\rangle_{X, Y}$ (note that, once the singlet state is created, it remains the singlet state in every polarization basis, so $\left.\left|\psi^{(-)}\right\rangle_{X, Y}=\left|\psi^{(-)}\right\rangle_{H, V}\right)$.

Our experimental setup is depicted in Fig. 1. The photon pairs were generated by two adjacent, 1 -mm-thick $\beta$-barium borate (BBO) crystals, pumped with a continuous argon laser $(180 \mathrm{~mW}$ at $363.8 \mathrm{~nm}$, polarized along the $X$ axis), and oriented to obtain collinear type-I down-conversion. By rotating the pump polarization, and by making sure the entire optical setup had the same attenuation for all the polarizations, we ensured that both the generation and the detection of the photon pairs occurred at the same probability for $V$ and $H$ polarizations. A computer-controlled liquid crystal cell (LCC), placed immediately after the two crystals, controlled the relative phase $\beta$ between the $H$-polarized pairs and the $V$-polarized ones. The photons were then directed to a phase and polarization pulse shaper [10], which included two orthogonal SLMs, one applying a spectral phase $\theta_{X}(\omega)$ to $X$-polarized photons, and the second applying a spectral phase $\theta_{Y}(\omega)$ to $Y$-polarized photons. The outcoming beam was then split by a polarizing beam splitter (PBS) to $H$ - and $V$-polarized beams, which were focused onto two singlephoton counting modules (SPCMs). The coincidence counts were recorded with a temporal resolution of $12.5 \mathrm{~ns}$.

A key point in this scheme is that the PBS at the output of the pulse shaper mixes the $X, Y$ polarizations, and thus induces a quantum interference between the two two-photon wave functions that contribute to coincidence detections in the SPCMs. Using the LCC, we set the phase $\beta$ to $\pi$, thus creating the state of Eq. (2) at the input of the pulse shaper, in which each SLM affected only one photon of the pair. This allowed us to induce a relative delay between the photons by simply applying linear spectral phases with opposite slopes on the two SLMs: $\theta_{X, Y}(\omega)= \pm \tau \omega$ [9]. This configuration is completely equivalent to the two-photon interference experiment by Hong, Ou, and Mandel (HOM) [11], and indeed, a scan of the relative delay between the photons reproduced the so-called HOM dip in the coincidence counts with a visibility of $0.79 \pm 0.01$ [Fig. 2(a)].

Since our setup enables the application of arbitrary phases to each of the spectral and polarization modes of the photons, it allows convenient exploration of the spectrally dependent quantum interference that governs the HOM interference pattern, which was previously explored only by utilizing interference filters or spectrally independent phase shifters [17]. In pulsed down-conversion, or with photons from independent single-photon sources, the HOM interference pattern depends on the overlap between the (independently defined) temporal envelopes of the two photons. This is not the case with time and energy entangled photons, which can exhibit a zero coincidence rate even if the photons do not overlap temporally at the beam splitter [18]. At low photon fluxes, the coincidence rate $R_{c}$ between the two output ports of the HOM interferometer is given by [19]

$$
R_{c} \propto \int_{T} d \tau\left|\left\langle 0\left|\hat{E}^{+}\left(\mathbf{r}_{2}, t+\tau\right) \hat{E}^{+}\left(\mathbf{r}_{1}, t\right)\right| \varphi\right\rangle\right|^{2}
$$

where $\mathbf{r}_{1}$ and $\mathbf{r}_{2}$ are the detector locations, $\tau$ is the time difference between the two detection events, and $T$ is the coincidence-circuit temporal resolution. Inserting $|\varphi\rangle$ from Eq. (2) into Eq. (4) and accounting for the spectral phases 

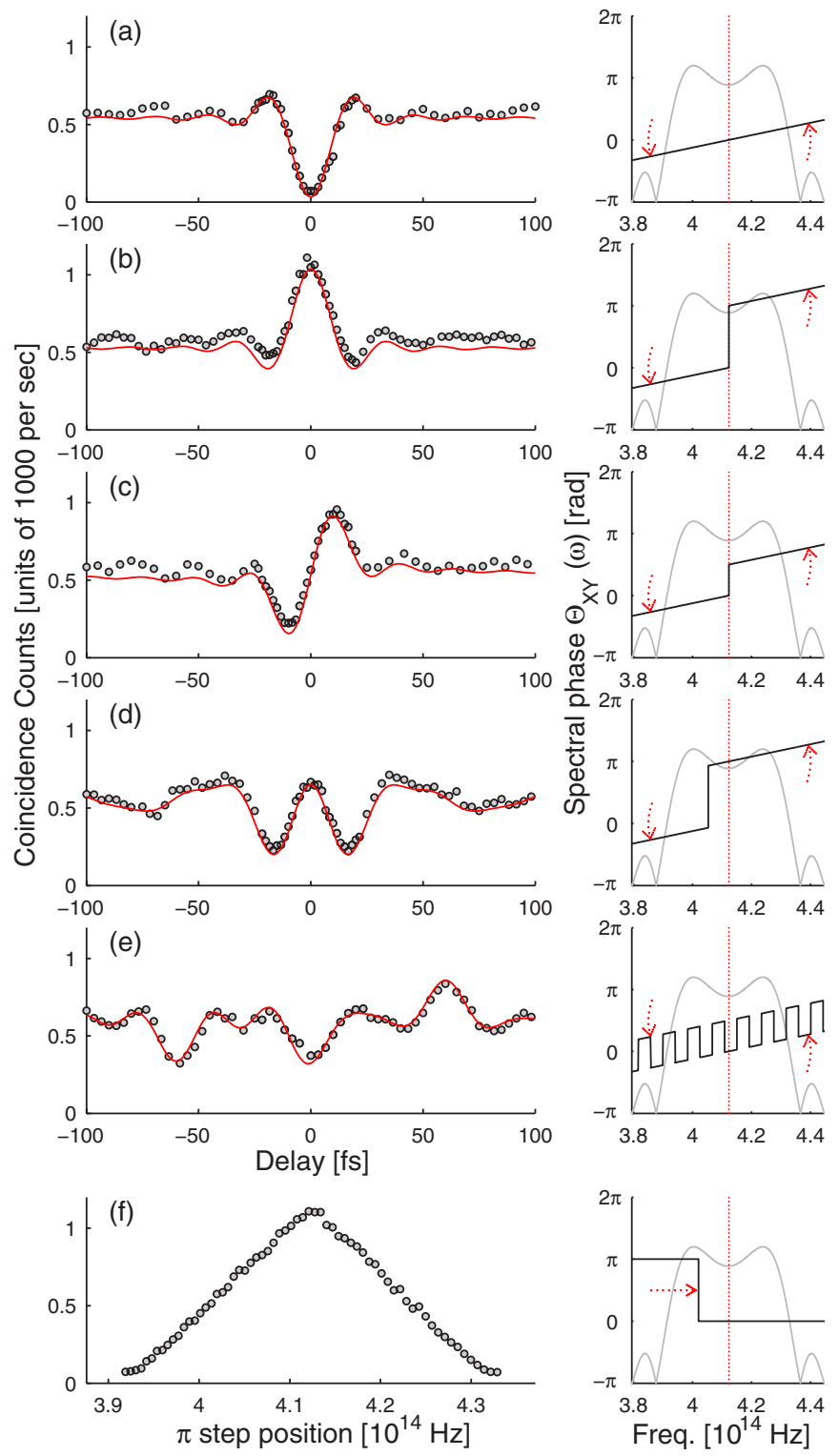

FIG. 2. (Color online) (a)-(e) Left column shows the experimental results (circles) and theoretical calculation (line) of the coincidence count $R_{c}$ vs the applied delay between the $X$ and $Y$ polarizations, for various spectral phase functions $\Theta_{X Y}(\omega)$, which are depicted in the right column (black), together with the downconverted spectrum (gray). (f) Same as (b), except that the delay is set to zero and the $\pi$-step position is scanned over the spectrum. The power spectrum used in the calculation of Eq. (5) was obtained by fitting the curve (f) to the integral of the power spectrum expected for type-I down-conversion. No other fitting procedures were applied.

applied by the SLMs, one gets the following expression for $R_{c}$ :

$$
R_{c} \propto\left(1-\int_{0}^{\omega_{0}} d v|g(v)|^{2} e^{\iota\left[\Theta_{X Y}(v)-\Theta_{X Y}(-v)\right]}\right),
$$

where

$$
\Theta_{X Y}(v) \equiv \theta_{X}\left(\omega_{0}+v\right)-\theta_{Y}\left(\omega_{0}+v\right)
$$

As evident from Eq. (6), only the spectral phase difference between the interferometer arms counts. Thus, when identical phase functions were applied by both SLMs, the delay scan yielded the same HOM dip as depicted in Fig. 2(a). For this reason, in Fig. 2 we presented our results as they are related to the spectral phase difference between the SLMs $\Theta_{X Y}(\omega)$. Moreover, Eq. (5) indicates that only a spectral phase difference that is antisymmetric with respect to $\omega_{0}$ affects the two-photon interference pattern [20]. This effect of nonlocal dispersion cancellation, which was previously predicted [21,22] and demonstrated [23] for first-order dispersion, was demonstrated in our setup for all odd orders of dispersion by the fact that applying arbitrary spectral phases whose difference was symmetric about $\omega_{0}$ had no effect on the HOM dip. Another interesting result of Eq. (5) is that the coincidence function $R_{c}$ could be zero only at one value of the delay between the interferometer arms; such a zero occurs if and only if there is no antisymmetric spectral phase difference between the interferometer arms, and then the shape of the HOM dip is dictated only by the power spectrum of the down-converted photons.

Figures 2(b)-2(e) demonstrate our ability to arbitrary tailor the shape of the HOM interference pattern, with excellent agreement with the theoretical calculation according to Eq. (5). It is interesting to note the difference between Figs. 2(a) and 2(b) at zero delay. The first occurs when the state of the photon pairs is essentially the "untouched" initial $\left|\phi^{(-)}\right\rangle_{H, V}$, which is composed only of two horizontal photons or two vertical ones, and therefore yields zero coincidences at the two ports of the PBS. The second occurs when a $\pi$ step function is applied by one of the SLMs, with the step located at the frequency $\omega_{0}$. In this case the state of the pairs becomes the singlet state $\left|\psi^{(-)}\right\rangle_{H, V}$, which always results in coincidences between the output ports of the PBS. Since the difference between the two states results from a destructive spectral interference which turns into a constructive one due to the presence of the spectral $\pi$ step function, we can induce this change gradually by scanning the position of the step function along the down-converted spectrum. The result of such a scan appears in Fig. 2(f). Since the coincidence rate is proportional to the relative part of the spectrum that experiences the phase flip, the actual down-converted power spectrum is the absolute value of the derivative of this graph. The power spectrum derived from Fig. 2(f) was used in the calculation of the theoretical curves in Figs. 2(a)-2(e).

Finally, we used the computer-controlled pulse shaper and LCC to generate the four polarization Bell states. By setting the phase $\beta$ to $\pi$ or 0 we generated the states $\left|\phi^{(-)}\right\rangle_{H, V}$ and $\left|\phi^{(+)}\right\rangle_{H, V}$, respectively. The remaining two Bell states were generated from these two states by applying the spectral $\pi$ step function, located at $\omega_{0}$ on one of the SLMs. As explained earlier, since at the principal axes $X, Y$ of the SLMs $\left|\phi^{(-)}\right\rangle_{H, V}$ is $\left|\psi^{(+)}\right\rangle_{X, Y}$, it is turned into $\left|\psi^{(-)}\right\rangle_{X, Y}=\left|\psi^{(-)}\right\rangle_{H, V}$ by such a phase step function. Similarly, $\left|\phi^{(+)}\right\rangle_{H, V}=\left|\phi^{(+)}\right\rangle_{X, Y}$ is turned into $\left|\phi^{(-)}\right\rangle_{X, Y}=\left|\psi^{(+)}\right\rangle_{H, V}$ by the same spectral phase function. In order to measure the generated Bell states we introduced a half-wave plate between the output port of the pulse shaper and the PBS, with its principal axis rotated by $\alpha / 2$ from the axis $H\left(0 \leq \alpha \leq 180^{\circ}\right)$. Thus, we measured for each state the coincidence counts rate $R_{\alpha, \alpha^{\perp}}$ at orthogonal 
polarizations $\alpha, \alpha^{\perp}$ which were rotated by the angle $\alpha$ with respect to $H, V$. Similarly, we measured the coincidence rate of two photons at the same polarization axis, namely, $R_{\alpha, \alpha}$. We did so by locating a polarizer at the angle $\alpha$ at the output port of the pulse shaper, and using the following half-wave plate to rotate this polarization to the axis $X$. By doing this, $\alpha$ polarized photons had an equal, independent probability to be directed to either of the SPCMs, which meant that the measured coincidence rate corresponded to half of the actual coincidence rate of $\alpha$ polarized photons. The experimental results are depicted in Fig. 3, together with the theoretical curves. This set of measurements provides sufficient information about the density matrix of the generated states to deduce lower bounds on their fidelities with the desired Bell states, indicating that the Bell states $\left|\psi^{(-)}\right\rangle_{H, V},\left|\psi^{(+)}\right\rangle_{H, V},\left|\phi^{(-)}\right\rangle_{H, V},\left|\phi^{(+)}\right\rangle_{H, V}$ were produced with fidelities that are equal to or greater than $0.90 \pm 0.01$, $0.88 \pm 0.01,0.85 \pm 0.02,0.84 \pm 0.01$, respectively. Let us also note that three out of the four generated states $\left(\left|\psi^{(-)}\right\rangle_{H, V}\right.$, $\left|\psi^{(+)}\right\rangle_{H, V}$, and $\left.\left|\phi^{(-)}\right\rangle_{H, V}\right)$ demonstrate nonclassical behavior by violating the Cauchy-Schwartz inequality for the secondorder correlation functions, with $R_{\alpha, \alpha} R_{\alpha^{\perp}, \alpha^{\perp}}<R_{\alpha, \alpha^{\perp}}^{2}$.

The ability to generate Bell states in a collinear fashion which allows coupling into an optical fiber bears great significance for quantum communication (see, for example, [14]). We believe that our scheme will allow efficient coupling of ultrahigh fluxes of entangled pairs into fibers, fluxes that are many orders higher than those achievable by pulsed systems [8]. Note that our scheme also enables the assignment of different Bell states to different spectral mode pairs, thus creating a quantum equivalent to wavelength-division multiplexing in classical optical communication systems. Additionally, the singlet state of this scheme, which appears to be the most robust DFS that can be supported in a singlemode fiber, could be used to conveniently transport indistinguishable photon pairs over large distances on the same fiber, and then separate them deterministically. This is due to the fact that any PBS located at the output of the fiber will separate the photons, one to each port, with no need to compensate for the (usually time-dependent) birefringence. Note that in this case the photons share the same spectrum, and are
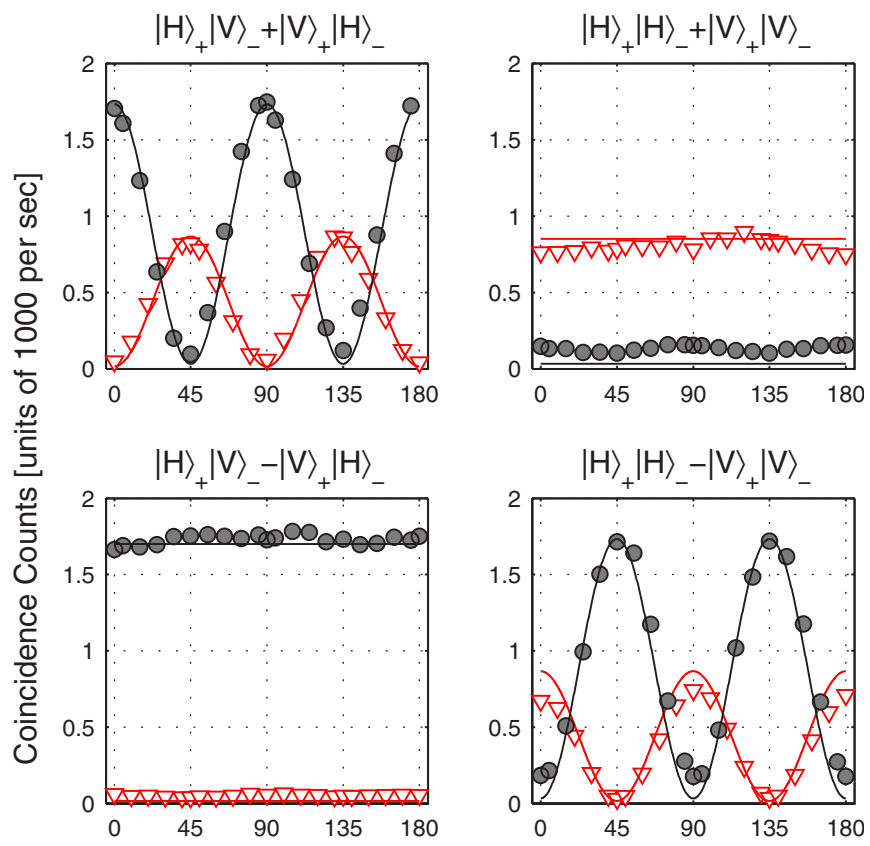

Analyzer axis angle $\alpha$ [deg]

FIG. 3. (Color online) Coincidence measurements illustrating the production of all four Bell states in our system. The plots show the experimental coincidence rates $R_{\alpha, \alpha^{\perp}}$ (circles) and $R_{\alpha, \alpha}$ (triangles) vs the angle of the analysis axis $\alpha$ with respect to $H$, as compared to the theoretical calculations (line) which assume pure Bell states. $R_{\alpha, \alpha^{\perp}}$ indicates coincidence of orthogonally polarized photons (at polarizations $\alpha, \alpha^{\perp}$ ), and $R_{\alpha, \alpha}$ indicates coincidences of photons that are both $\alpha$ polarized. The visibilities of the $\left|\psi^{(+)}\right\rangle_{H, V}$ $\left(\left|\phi^{(-)}\right\rangle_{H, V}\right)$ state are $0.90 \pm 0.02(0.93 \pm 0.01)$ for the $R_{\alpha, \alpha}$ curve and $0.90 \pm 0.01(0.81 \pm 0.02)$ for the $R_{\alpha, \alpha^{\perp}}$ curve.

time and energy entangled. These abilities could become useful in various quantum communication and quantum cryptography schemes [13].

The authors wish to thank Avi Pe'er for many fruitful discussions. Financial support of this research by the Israel Science Foundation is gratefully acknowledged.
[1] K. Mattle, H. Weinfurter, P. G. Kwiat, and A. Zeilinger, Phys. Rev. Lett. 76, 4656 (1996); D. Bouwmeester et al., Nature (London) 390, 575 (1997); T. Jennewein, C. Simon, G. Weihs, H. Weinfurter, and A. Zeilinger, Phys. Rev. Lett. 84, 4729 (2000); D. S. Naik, C. G. Peterson, A. G. White, A. J. Berglund, and P. G. Kwiat, ibid. 84, 4733 (2000).

[2] P. G. Kwiat, K. Mattle, H. Weinfurter, A. Zeilinger, A. V. Sergienko, and Y. Shih, Phys. Rev. Lett. 75, 4337 (1995); Y.-H. Kim, S. P. Kulik, and Y. Shih, Phys. Rev. A 63, 060301(R) (2001); C. Kurtsiefer, M. Oberparleiter, and H. Weinfurter, ibid. 64, 023802 (2001); M. Barbieri, F. DeMartini, G. Di Nepi, and P. Mataloni, Phys. Rev. Lett. 92, 177901 (2004); M. Fiorentino, G. Messin, C. E. Kuklewicz, F. N. C. Wong, and J. H. Shapiro, Phys. Rev. A 69, 041801(R) (2004).
[3] P. G. Kwiat, E. Waks, A. G. White, I. Appelbaum, and P. H. Eberhard, Phys. Rev. A 60, R773 (1999).

[4] A. V. Burlakov, M. V. Chekhova, O. A. Karabutova, and S. P. Kulik, Phys. Rev. A 64, 041803(R) (2001).

[5] P. G. Kwiat et al., Science 290, 498 (2000).

[6] W. A. T. Nogueira, S. P. Walborn, S. Padua, and C. H. Monken, Phys. Rev. Lett. 92, 043602 (2004).

[7] J. Javanainen and P. L. Gould, Phys. Rev. A 41, 5088 (1990).

[8] B. Dayan, A. Peer, A. A. Friesem, and Y. Silberberg, Phys. Rev. Lett. 94, 043602 (2005).

[9] A. Pe'er, B. Dayan, A. A. Friesem, and Y. Silberberg, Phys. Rev. Lett. 94, 073601 (2005).

[10] T. Brixner and G. Gerber, Opt. Lett. 26, 557 (2001).

[11] C. K. Hong, Z. Y. Ou, and L. Mandel, Phys. Rev. Lett. 59, 
2044 (1987).

[12] P. G. Kwiat, J. Mod. Opt. 44, 2173 (1997); M. Barbieri, C. Cinelli, P. Mataloni, and F. De Martini, Phys. Rev. A 72, 052110 (2005); J. T. Barreiro, N. K. Langford, N. A. Peters, and P. G. Kwiat, Phys. Rev. Lett. 95, 260501 (2005).

[13] N. Gisin et al., Rev. Mod. Phys. 74, 145 (2002).

[14] K. Banaszek, A. Dragan, W. Wasilewski, and C. Radzewicz, Phys. Rev. Lett. 92, 257901 (2004).

[15] Or any other antisymmetric division of the spectrum.

[16] A. M. Weiner, Rev. Sci. Instrum. 71, 1929 (2000).

[17] Z. Y. Ou and L. Mandel, Phys. Rev. Lett. 61, 54 (1988); J. G. Rarity and P. R. Tapster, ibid. 64, 2495 (1990); H. Kim, J. Ko, and T. Kim, J. Opt. Soc. Am. B 20, 760 (2003); Y. J. Lu, R. L. Campbell, and Z. Y. Ou, Phys. Rev. Lett. 91, 163602 (2003);
A. Zavatta, S. Viciani, and M. Bellini, Phys. Rev. A 70, 023806 (2004); R. Okamoto, S. Takeuchi, and K. Sasaki, ibid. 74, 011801(R) (2006).

[18] T. B. Pittman, D. V. Strekalov, A. Migdall, M. H. Rubin, A. V. Sergienko, and Y. H. Shih, Phys. Rev. Lett. 77, 1917 (1996).

[19] L. Mandel and E. Wolf, Optical Coherence and Quantum Optics (Cambridge University Press, New York, 1995).

[20] W. P. Grice and I. A. Walmsley, Phys. Rev. A 56, 1627 (1997). [21] J. D. Franson, Phys. Rev. A 45, 3126 (1992).

[22] A. M. Steinberg, P. G. Kwiat, and R. Y. Chiao, Phys. Rev. A 45, 6659 (1992).

[23] A. M. Steinberg, P. G. Kwiat, and R. Y. Chiao, Phys. Rev. Lett. 68, 2421 (1992). 